\title{
New Optimized Crossover Utilizes Exponential Monte Carlo
}

\author{
Mufid Mohamed A. Musrati and Hanan Ettaher Dagez
}

\begin{abstract}
Crossover operator proved to be very important component for supporting diversity strategy, and producing new population. Many researches came out with different operators that are suitable to different types of problems. However, some of crossover techniques usually produce infeasible solution which implies the use of repair function to rebuild the solution. Moreover, some of existing crossover operators is not suitable for high constrained problems. Course timetabling of university is known to be highly constrained optimization problem, where lectures assigned to specific timeslots and rooms, with maintaining feasibility and satisfying soft constraints. In this paper, we present genetic algorithms for solving course timetabling problem. The algorithm consists of new optimized crossover utilizes Exponential Monte Carlo with Counter. The approach is tested and the result demonstrates that our approach is able to produce good quality solutions for course timetable problem.
\end{abstract}

Index Terms-Genetic algorithms, crossover, course timetabling

\section{INTRODUCTION}

There had been various attempts to automate university timetabling system using various methods. Timetabling problems occur in various different areas including transportation, sports events, employee and university [1]. Timetabling problems are highly constrained and difficult to solve this optimality. It consist of assigning number of events (course lectures) to limited number of timeslots. Constraints are usually classified into hard constraints and soft constraints Hard constraints cannot be violated and soft constraints are not essential to be satisfied but are desirable. This research focuses on course timetabling problems [2] which consists of the following:

- Set of $\mathrm{n}$ events $\mathrm{E}$ to be scheduled in;

- Set of timeslots $\mathrm{T}=45$

- Set of rooms $\mathrm{R}$ in which events can take place

- Set of students $\mathrm{S}$ who attend the events

- Set of features F satisfied by rooms and required by events.

Each student attends a number of events and each room has a maximum capacity. A feasible timetable is one in which all events have been assigned a timeslot and a room so that the following hard constraints are satisfied:

- No student attends more than one event at the same time.

Manuscript received February 15, 2014; revised April 28, 2014.

Mufid Mohamed A. Musrati is with High Institute of Industrial Technology Engila, Tripoli (e-mail: mufid12@yahoo.com).

Hanan Ettaher Dagez is with Faculty of IT, University of Tripoli, Libya (e-mail: h.dagez@it.uot.edu.ly).
- Room size satisfies the student number and all the required features for the event.

- Only one room assigned to timeslot for each event.

Feasible candidate timetable is penalized equally for each occurrence of the following soft constraint violations:

- Student has a class in the last timeslot of the day.

- Student has more than two classes in a row for one day.

- Student has only one class a day.

Genetic algorithm is one of the techniques being used to solve course timetabling problem. GA has been widely used in many areas that are difficult for humans to handle. The solutions are more credible, efficient, faster, and of higher complexity than humans can solve [3]. Genetic algorithm employs several operators such as: selection, crossover and mutation. These operators manipulate different number of solutions to improve the fitness function. Crossover operator is used to create child from existing parents and responsible for the propagation of the characteristics of the fittest individuals.

There are three kind of basic crossover which is single point, two point and uniform crossover. [4] discussed the crossover operation of one point, two points and uniform crossover techniques, and argued that feature of this methods is that the crossover points are determined randomly, and the resulting child is created without regard to the objective function.

Some of existing crossover operators are usually not suitable for high constrained problems where they usually produce infeasible solution [3]. Moreover, they do not utilize fitness function to improve the child solution [5].

There are many attempts to improve crossover operator to solve constraint satisfaction problems. These attempts provide good results for some problem, but still unsatisfied for high constrained problems such as course timetable problem. Even though, the optimized crossover approach in the research [5] and [6] were capable to produce good result for some problem. Moreover, their crossover approach exploits the structure of the solution rather than its coding which ease the search for good result solutions. One research has applied optimized crossover within genetic algorithm and produced two new children: The Optimum child (O-child) and Exploratory child (E-child) [4].

The O-child is constructed in such a way that has the best objective function value from a feasible set of children, while the E-child is constructed so as to maintain the diversity of the search space [7]. However, these approaches still need improvement due to the following drawback:

1) Usually produces infeasible solution which implies the use of repair function.

2) The created children seemed does not inherit adequate 
information from its parents, (i.e. just few variables can be posted from parents to children due to hard constraints imposed).

3) The utilization of very large neighborhood local search certainly will lead the search to stuck in local optima. Therefore, our research approach is New Optimized Crossover (NOX). NOX is knowledge-based crossover where new children produced using optimization routine searching for the best objective function. NOX approach viewed as a very large-scale neighborhood search.

In order to counter the drawback of literature-optimized crossover, heuristics for NOX proposed and MECQ criteria adopted for NOX.

\section{NEW OPTIMIZED CROSSOVER (NOX)}

NOX starts by copying parent2 (p2) as child1 (C1) and copying parent1 (P1) as child2 (C2). Child1 crossover is started by posting the timeslots of $\mathrm{P} 1$ events into the equivalent $\mathrm{C} 1$ events. Checking for the hard constraint (student courses conflict) violation applied and if so, the replacement not accepted and other P1event timeslot posted to the equivalent event of $\mathrm{C} 1$ event. If no violation to student courses conflict, then checking to room conflict hard constraint applied and if so, solve this violation by moving the original event timeslot of $\mathrm{C} 1$ to the event that has conflict with it. Check for student courses conflict, which may arise from the last movement. If there is conflict in student courses cancel the previous movement and post Plevent timeslot which equivalent to $\mathrm{C} 1$ event and check for student courses and room conflicts. If there is no violation to the hard constraints check the fitness function value of the child. If no violation to the hard constraints then, check the fitness function value. If it is equal (the new solution might be different) or less than the best solution. In child 2 crossover, worst solution might be accepted based on EMCQ criteria. Based on our preliminary tests, the crossover rate is set to (0.9). Fig. 1 illustrated example for NOX to solve course timetable problem. Acceptance criteria applied for the worst solution is to maintain the diversity of the search space and to escape from local optima. Exponential Monte Carlo with counter (EMCQ) is used to accept worse solution with a certain probability [8].

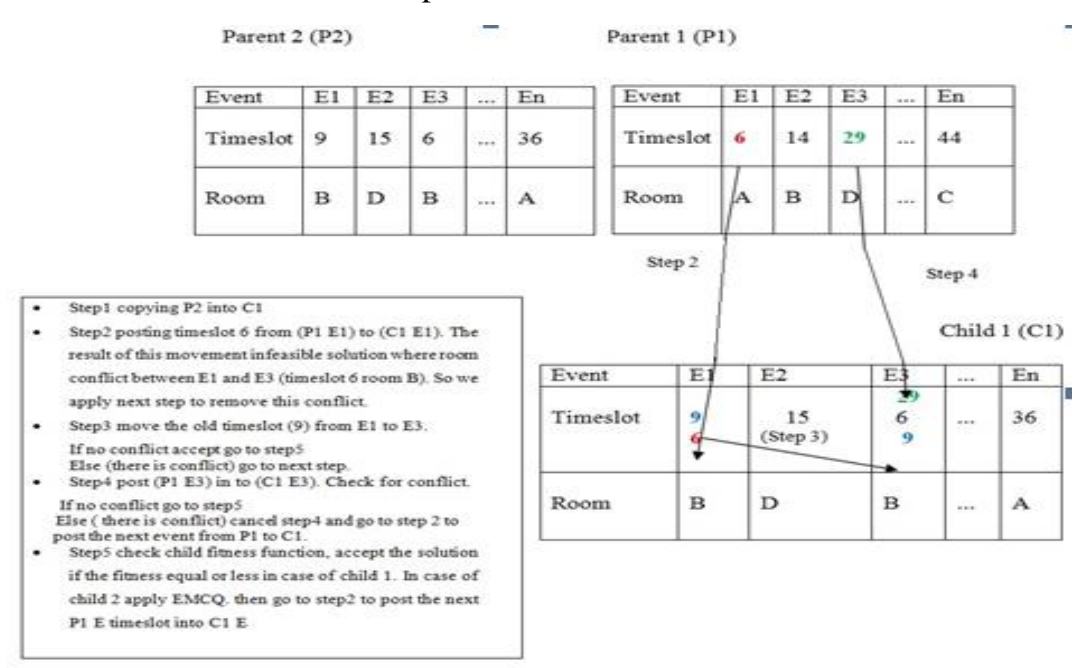

Fig. 1. An example of NOX.

\section{EXPERIMENTS}

Experiment on population size that monitors the behavior of the GA as the population size alters. To determine the appropriate population size, we ran 10 tests on medium instance. The iteration number for the approach is 400000 .

In this experiment we noted that when the population size less than (50) chromosomes iterates very rapidly and converges to a solution very quickly. But the penalty value significantly higher due to the lack of diversity.

Large populations (i.e. more than 50) are carrying many different individuals, but takes longer time to converge to good quality solution.

Population size (50) gives better solution and after monitoring the fitness value and time taken to converge to the best solution for each population size, population size of 50 chromosomes have been implemented due to the best result gained from this population size where it characterized by lower penalty value and shorter time taken to converge to the best solution. Table I, below lists the result of this experiment.

\begin{tabular}{|c|c|c|c|}
\hline TABLE I: POPULATION SIZE EXPERIMENT \\
\hline Population size & $>50$ chroms. & 50 chroms. & $>50$ chroms. \\
\hline $\begin{array}{c}\text { The best fitness } \\
\text { function value }\end{array}$ & 470 & 371 & 484 \\
\hline
\end{tabular}

The next experiments are investigate the use of two types of Monte Carlo acceptance criteria on NOX, these are Exponential Monte Carlo (EMC) and Exponential Monte Carlo with counter (EMCQ). We also investigate the use of accepting worst solution in one of the child (i.e. in child1 crossover we accept improved movement only and in second child 2 crossover we accept worst movement) and in both two children (i.e. worst movement accepted in both child crossover). The best result obtained out of 10 runs on dataset medium1.

Fig. 2 shows the performances of NOX using EMC to one child only. While on the other child crossover, we accept only improved or equal solution. 

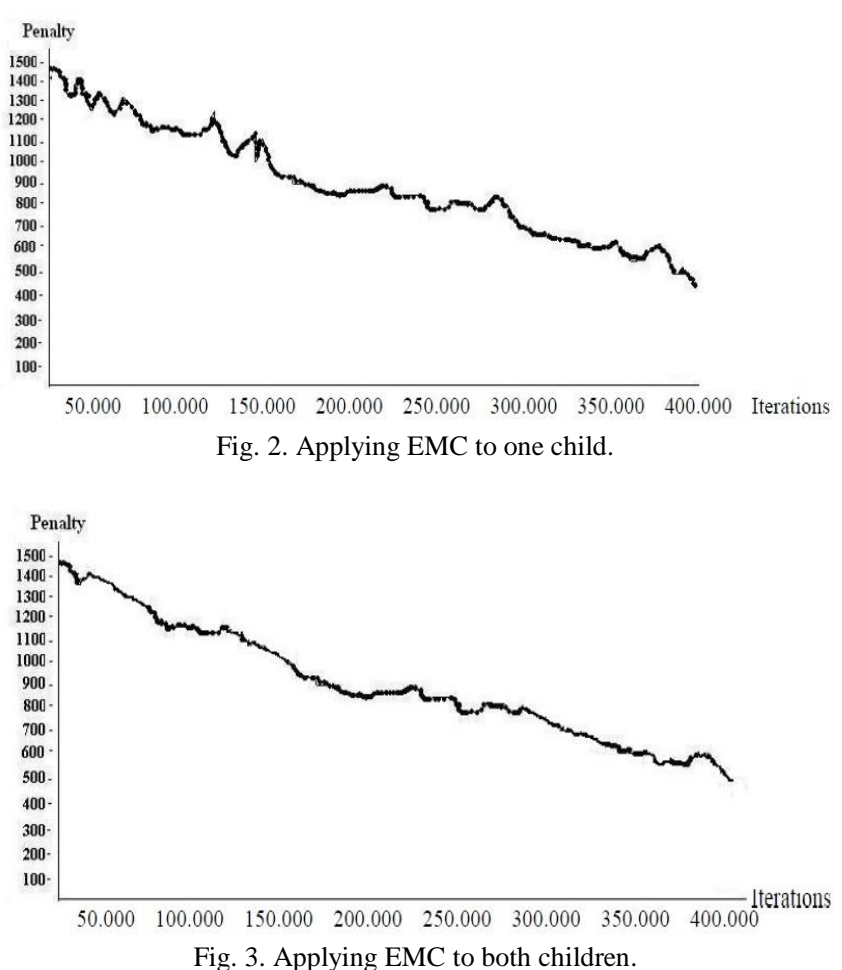

The slope of curves in Fig. 3, indicates that the search takes long time to converge to the best solution, where it reaches the best solution (450) penalty cost at the end of the (400000) iterations.

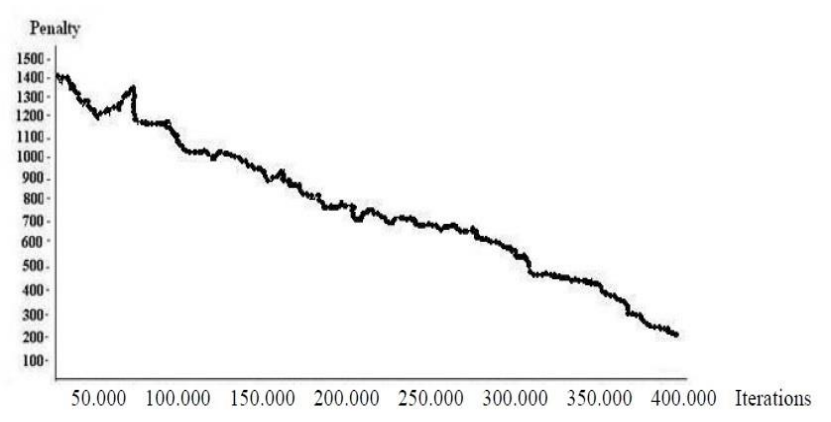

Fig. 4. Applying EMCQ to two children.

Fig. 4 shows the effect of applying EMCQ. In this approach, the improved solution is always accepted and worst solution will be accepted based on probability that depends on solution quality and computation time.

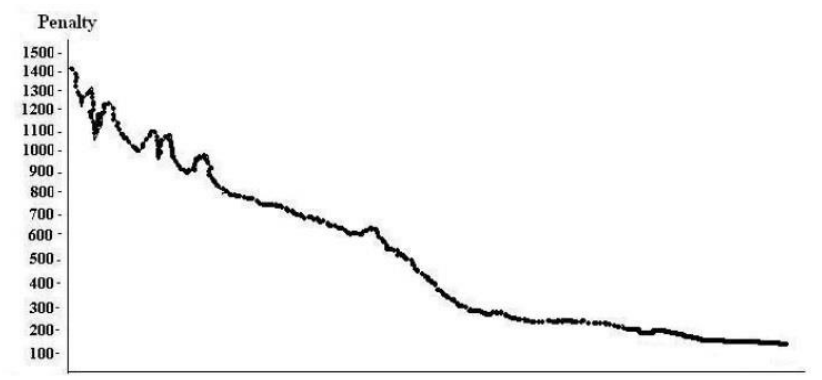

$\begin{array}{llllllll}50.000 & 100.000 & 150.000 & 200.000 & 250.000 & 300.000 & 350.000 & 400.000\end{array}$ Iterations

Fig. 5. Applying EMCQ to one child.

EMCQ is applied for both children and the result shown in Fig. 5, the best result is (300) penalty cost at the end of (400000) iterations.
Fig. 5 shows the performances of the NOX where EMCQ applied to one child only and the acceptance of the improved or equal solution only applied to the other child. The slope of curves indicates the improvement of the solution up to (271) penalty cost at the iterations (300000). The analysis of Fig. 3, and Fig. 4, showed that the use of EMC in one child crossover only, Fig. 3, provided better quality solution than the use of EMC in both children Fig. 4. We also noticed during the analysis in Fig. 4, and Fig. 5, that the use of EMCQ in one child crossover only gives better quality solution than use of EMCQ in both children. Therefore, the acceptance of worst solution criteria techniques (EMC or EMCQ) when applied in one child crossover only tend to support the diversity of solutions in one child and to support intensifying the solutions in the other child crossover. Thus, applying EMCQ to one child only and applying the acceptance of the improved solution only to other child increases the ability to search more space and reduce the time to reach the better solutions. The analysis of the all diagrams showed that the penalty cost can be quickly reduced as illustrated in all the figures, until the middle of the diagrams (between iterations 200000 and 250000). Fig. 2, and Fig. 3, (where EMC criteria employed) show slow improvement in the solution quality started from the end of iteration 250000 while Fig. 4, and Fig. 5, showed continuing improvement of the solution quality. Moreover, the highest penalty value solutions at the end of iterations were showed in Fig. 2 and Fig. 3. Fig. 4 and Fig. 5 showed the best quality solution. Therefore, we believe that EMCQ is better than EMC because EMC criteria only based on the quality of the solution, but in the EMCQ criteria the factor of time is included as an intensification factor. (i.e. worse solution is more likely to be accepted but as the time increases the worse solution is unlikely to be accepted).

Table II shows the comparison of our approaches with graph hyper heuristic by Burke et al. (2007), and hybrid approach by Abdullah et al. (2008). The comparison with hyper heuristic and hybrid approaches is to evaluate our approach solution quality against other optimization techniques. The constructive heuristic employed was unable to produce a feasible solution for large dataset.

TABLE II: COMPARISON RESUlT ON COURSE TIMETABLING PROBLEM

\begin{tabular}{|l|l|l|l|l|c|}
\hline \multirow{2}{*}{ Dataset } & \multicolumn{2}{|c|}{$\begin{array}{c}\text { Our approach GA } \\
\text { using } \\
\text { NOX with EMCQ }\end{array}$} & $\begin{array}{l}\text { Abdullah } \\
\text { et al. 2008 }\end{array}$ & $\begin{array}{c}\text { Burke } \\
\text { et al. } 2007\end{array}$ \\
\cline { 2 - 6 } & Best & Ave & Best & Ave & Best \\
\hline small 1 & 8 & 9 & 0 & 0 & 6 \\
\hline small2 & 9 & 10 & 0 & 0 & 7 \\
\hline small3 & 8 & 9 & 0 & 0 & 3 \\
\hline small4 & 8 & 10 & 0 & 0 & 3 \\
\hline small5 & 9 & 11 & 0 & 0 & 372 \\
\hline $\begin{array}{l}\text { medium } \\
1\end{array}$ & 371 & 376 & 236 & 240.2 & 419 \\
\hline $\begin{array}{l}\text { medium } \\
2\end{array}$ & 366 & 378 & 158 & 160 & 359 \\
\hline $\begin{array}{l}\text { medium } \\
3\end{array}$ & 373 & 375.6 & 261 & 262.6 & 348 \\
\hline $\begin{array}{l}\text { medium } \\
4\end{array}$ & 357 & 359.4 & 176 & 178.5 & 171 \\
\hline $\begin{array}{l}\text { medium } \\
5\end{array}$ & 263 & 266 & 147 & 149.5 & \\
\hline large & $100 \%$ inf & 1227.8 & 296 & 315 & 1068 \\
\hline
\end{tabular}




\section{CONCLUSION}

Crossover operator is very important component to explore the diversity of search space. There are many attempts to improve crossover to produce child that inherit the useful information from the parents. This paper introduces the advantages and disadvantages of the available crossover techniques. Although the analysis of the optimized crossover approach shows that it can produce a good result. However, still the search to get the optimal solution is going on. Therefore, this research is attempt to improve the optimized crossover approach by introduce new optimized crossover technique.

\section{REFERENCES}

[1] V. A. Bardadym, "Computer aided school and university timetabling: The new wave, in practice and theory of automated timetabling," in Proc. the 1st International Conference on the Practice and Theory of Automated Timetabling, Edinburgh, August/September 1995, vol. 1153 , pp. 22-45.

[2] K. Socha, J. Knowles, and M. Samples, "A max-min ant system for the university course timetabling problem," in Proc. the 3rd International Workshop on Ant Algorithms, Lecture Notes in Computer Science 2463, 2002, pp. 1-13.

[3] O. I. Obaid, M. Ahmad, S. A. Mostafa, and M. A. Mohammed, Comparing Performance of Genetic Algorithm with Varying Crossover in Solving Examination Timetabling Problem.

[4] C. Meyers and J. B. Orlin, "Very large-scale neighborhood search techniques in timetabling problems," in Proc. the 6th International Conference on Practice and Theory of Automated Timetabling, Berlin, 2007, pp. 24-39.

[5] Journal of Emerging Trends in Computing and Information Sciences, vol. 3, no. 10, Oct 2012.

[6] C. C. Aggarwal, J. B. Orlin, and R. P. Tai, "Optimized crossover for the independent set problem," Operation Research, vol. 45, issue 2, March-April 1997.
[7] H. Nazif and L. S. Lee, "Optimized crossover genetic algorithm for vehicle routing problem with time windows," American Journal of Applied Sciences, vol. 7, no. 1, pp. 95-101, 2010.

[8] M. Ayob, "Optimisation of surface mount device placement machine in printed circuit board assembly," $\mathrm{PhD}$ Thesis, University of Nottingham, UK, 2006.

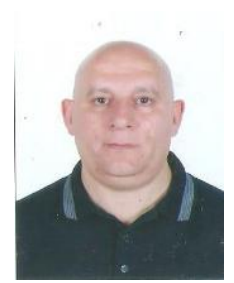

Mufid Mohamed A. Musrati was born in 1964 in Tripoli, Libya. He obtained postgraduate diploma in computer science from University of Malaya, Malaysia, 2000. In 1989, he got certificate as aircraft maintains engineer.

His working experience started since 1990 to 1995 as aircraft maintenance engineer. Since 2010 to 2014 working as an instructor in Computer Department and as supervisor for number of graduation projects at Engila Higher Institute. Teaching and instructing IC3 program for ministry of labor and rehabilitation.

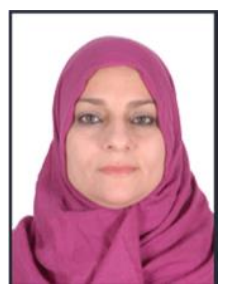

Hanan Ettaher Dageez was born in 1969 in Tripoli, Libya. She received her Ph.D. degree in computer science in field of e-learning from University of Malaya in 2010, master degree in field of software engineering project management from University of Malaya in 1999, bachelor degree in computer science from University of Tripoli (1992).

Her working experience started in 1992 as an instructor and since 1999 as a lecturer. During her career she was elected to be the head of Computer Department, and the head of Information Science Department and member of examination control committee member in Libyan Education Ministry. In 2009, she took part time lecturer in University of Malaya. Currently, she is working as a lecturer and the head of Department at Faculty of Information Technology in University of Tripoli, Libya. 